\title{
Compuestos sintéticos del tipo de estirilquinolinas con actividades leishmanicida y citotóxica
}

\author{
Emy Luz Sánchez¹, Gílmar Gabriel Santafé1, Omar Leonardo Torres², \\ Diana Lorena Muñoz ${ }^{3}$, Sara María Robledo ${ }^{3,4}$ \\ 1 Grupo de Investigación en Química de los Productos Naturales, Facultad de Ciencias Básicas, Universidad de \\ 2 Grupo de Investigación en Desarrollo y Evaluación de Fármacos y Afines, IDEFARMA, Facultad de Ciencias de \\ ${ }^{3}$ Programa de Estudio y Control de Enfermedades Tropicales, PECET, Instituto de Investigaciones Médicas,
} Córdoba, Montería, Colombia la Salud, Universidad de Córdoba, Montería, Colombia Facultad de Medicina, Universidad de Antioquia, Medellín, Colombia

${ }^{4}$ Centro para el Desarrollo de Productos, CIDEPRO, Medellín, Colombia

Introducción. La leishmaniasis es uno de los principales problemas de salud pública en muchos países, entre ellos, Colombia. Su tratamiento presenta dificultades, como la toxicidad de los medicamentos utilizados, la aparición de cepas resistentes y su duración prolongada con costos elevados, lo que justifica la búsqueda de nuevas alternativas terapéuticas.

Objetivo. Evaluar las actividades leishmanicida y citotóxica de tres compuestos del tipo de la 2-estirilquinolina: el 2-[(E)-2-(2,3-diacetiloxifenil)etenil]quinolin-8-il-acetato, E1; el 2-[(E)-2-(4-acetiloxi3-metoxifenil)etenil]quinolina, E2, y el 2-[(E)-2-(2,3-diacetiloxifenil)etenil]quinolina, E3.

Materiales y métodos. Las estirilquinolinas se obtuvieron por síntesis orgánica mediante reacción de condensación del tipo de Perkin a partir de 8-hidroxiquinaldina o quinaldina y aldehídos aromáticos. La actividad leishmanicida se evaluó en Leishmania (Viannia) panamensis mediante citometría de flujo y la citotoxicidad en las células U-937 mediante el micrométodo enzimático con MTT. Los resultados se expresaron como concentración letal media $\left(\mathrm{CL}_{50}\right)$ para la citotoxicidad y como concentración efectiva media $\left(\mathrm{CE}_{50}\right)$ para la actividad leishmanicida, calculadas con el método Probit.

Resultados. El compuesto E3 de la 2-estirilquinolina demostró una gran actividad en Leishmania (Viannia) panamensis. Se calculó un valor de $\mathrm{CE}_{50}$ de $1,4 \mu \mathrm{g} / \mathrm{ml}$ y un índice de selectividad de 3,9; los compuestos $\mathrm{E} 1$ y E2 presentaron valores de $\mathrm{CE}_{50}$ mayores de 5,6 y $68,1 \mu \mathrm{g} / \mathrm{ml}$, respectivamente. En cuanto a la citotoxicidad, se calcularon valores de $\mathrm{CL}_{50}$ desde 5,4 hasta $68,1 \mu \mathrm{g} / \mathrm{ml}$. El compuesto E2 resultó ser moderadamente tóxico, con una $\mathrm{CL}_{50}$ muy similar a la de la anfotericina $\mathrm{B}$, sustancia usada como control de citotoxicidad.

Conclusión. A pesar de su toxicidad, el compuesto E3 de la 2-estirilquinolina demostró una actividad prometedora contra $L$. $(V$.) panamensis, pues fue capaz de inhibir de manera significativa los amastigotes en el interior de la célula y de reducir la infección.

Palabras clave: Leishmania panamensis, leishmaniasis, quinaldinas.

doi: http://dx.doi.org/10.7705/biomedica.v34i4.2299

\section{Styrylquinolines-type synthetic compounds with leishmanicidal and cytotoxic activities}

Introduction: Leishmaniasis is a major public health problem faced by many countries, including Colombia. Its treatment has limitations such as the toxicity of the drugs used, the emergence of resistant strains, and prolonged and expensive treatments. Thus, there is an urgent need to find alternative solutions.

Objective: To evaluate the leishmanicidal and cytotoxic activities of three 2-styrylquinolines type compounds: 2-[(E)-2-(2,3-diacetyloxyphenyl)ethenyl]quinolin-8-yl-acetate, E1; 2-[(E)-2-(4-acetyloxy-3methoxyphenyl)ethenyl] quinoline, E2, and 2-[(E)-2-(2,3-diacetyloxyphenyl)ethenyl] quinoline, E3.

Materials and methods: The 2-styrylquinolines were obtained by organic synthesis using Perkintype condensation reaction from 8-hydroxy quinaldine or quinaldine and aromatic aldehydes. The leishmanicidal activity was evaluated on intracellular amastigotes of Leishmania (Viannia) panamensis

\section{Contribución de los autores:}

Emy Luz Sánchez: síntesis e identificación de derivados quinolínicos

Omar Leonardo Torres y Gílmar Gabriel Santafé: escritura y revisión del artículo

Sara Robledo: diseño y ejecución de pruebas biológicas de actividad citotóxica y leishmanicida, análisis de resultados

Diana Lorena Muñoz: diseño y ejecución de pruebas biológicas de las actividades citotóxica y leishmanicida 
by flow cytometry. The results were expressed as lethal concentration $50\left(\mathrm{LC}_{50}\right)$ for cytotoxicity and effective concentration $50\left(\mathrm{EC}_{50}\right)$ for leishmanicidal activity, calculated by the Probit method.

Results: $E 3$ showed high activity against $L$. (V) panamensis with a calculated $E_{50}$ value of $1.4 \mu \mathrm{g} / \mathrm{ml}$, and a selectivity index of 3.9; $\mathrm{E} 1$ and $\mathrm{E} 2$ showed higher $\mathrm{EC}_{50}$ values of 5.6 and $68.1 \mu \mathrm{g} / \mathrm{ml}$, respectively. For cytotoxicity, $\mathrm{LC}_{50}$ values ranging from 5.4 to $68.1 \mathrm{\mu g} / \mathrm{ml}$ were calculated. E2 was moderately toxic, showing an $\mathrm{LC}_{50}$ very similar to that of amphotericin $\mathrm{B}$, a substance used as cytotoxic control.

Conclusion: The styrylquinoline E3 is a promising compound against $L$. (V) panamensis, as it was able to significantly inhibit amastigotes inside the cell, reducing infection despite its toxicity.

Key words: Leishmania panamensis, leishmaniasis, quinaldines.

doi: http://dx.doi.org/10.7705/biomedica.v34i4.2299

La leishmaniasis es una enfermedad de amplia distribución mundial que pertenece al grupo de enfermedades originadas por la infección con protozoarios del género Leishmania, el cual incluye numerosas especies con la misma morfología, pero con comportamientos biológicos e inmunológicos diferentes, por lo que las características clínicas y la distribución geográfica de la enfermedad también son diversas. En América, las especies de Leishmania son transmitidas por la picadura de insectos del género Lutzomyia (1-3). La leishmaniasis puede ser cutánea, mucosa o visceral según afecte el tejido cutáneo, las mucosas o los órganos del sistema mononuclear fagocítico. La leishmaniasis cutánea, causada principalmente por parásitos del complejo Viannia, es la más predominante $(4,5)$.

La Organización Mundial de la Salud estima que la incidencia anual de casos de leishmaniasis cutánea está entre 1 y 1,5 millones (6). La leishmaniasis es endémica en 99 países de Asia, África, Europa, Oceanía y América, con una prevalencia de 12 millones de casos, y se calcula que unos 350 millones de personas están en riesgo. En Colombia, la leishmaniasis representa un problema de salud pública que se ha incrementado de manera significativa durante los últimos años. Se estima que en el país existen alrededor de 11 millones de personas en riesgo de contraer la enfermedad, principalmente en el área rural, y hasta la semana 22 del 2014 (25 al 31 de mayo), se habían confirmado 4.345 casos, de los cuales 4.292 correspondían a leishmaniasis cutánea $(7,8)$.

Actualmente, en el tratamiento de las diferentes formas de leishmaniasis se emplean compuestos de antimonio pentavalente y otras sustancias como

\footnotetext{
Correspondencia:

Gílmar Santafé Patiño, Departamento de Química, Universidad de Córdoba, Carrera 6 № 76-103, Montería, Colombia Teléfono: (313) 5690794

gsantafe@correo.unicordoba.edu.co

Recibido: 28/04/14; aceptado: 15/08/14
}

el isotianato de pentamidina y la miltefosina. Además de ser costosos, las dosis prescritas de estos medicamentos tienen una considerable toxicidad y deben usarse por tiempo prolongado, factor este que facilita el abandono del tratamiento $y$, por ende, la aparición de cepas resistentes $(9,10)$. En consecuencia, la búsqueda de nuevos medicamentos, ya sea a partir de productos naturales u obtenidos por procesos de síntesis orgánica, constituye una prioridad.

En este contexto, los compuestos quinolínicos son una unidad estructural de gran importancia en el tratamiento de las enfermedades protozoarias. Estas sustancias fueron aisladas por primera vez de la quina, extraída de la corteza del árbol de la cinchona, la cual se emplea desde la antigüedad para combatir la malaria, y se encuentran en una gran variedad de moléculas naturales y sintéticas que actúan selectivamente frente a algunos protozoos (11-14).

El sistema quinolínico hace parte de la estructura de las 2-estirilquinolinas (quinolinas 2 sustituidas), las cuales se han reportado como sustancias que exhiben una amplia gama de actividades farmacológicas, entre las que se destaca la actividad contra los protozoos (15). Las quinolinas 2 sustituidas se han catalogado como moléculas activas contra Leishmania amazonensis y Trypanosoma cruzi, al igual que contra Plasmodium vinckei petteri $(14,15)$; específicamente, se ha reportado su actividad contra Leishmania (Viannia) panamensis (15), L. donovani (16), L. amazonensis y L. infantum (12). También se conocen reportes de la actividad de algunos de estos compuestos en la inhibición del HIV, razón por la cual se consideran como agentes antivirales promisorios por su capacidad de inhibir, tanto in vitro como in vivo, la actividad de la enzima que conforma el material genético del virus en la célula $(17,18)$.

En esta investigación se reportan los resultados de la evaluación de la actividad leishmanicida en amastigotes intracelulares de $L$. (V.) panamensis 
(cepa UA140-pIReGFP) y la actividad citotóxica in vitro en la línea celular promonocítica humana U-937 para las estirilquinolinas 2-[(E)-2-(2,3diacetiloxifenil)etenil]quinolin-8-il-acetato (E1); 2-[(E) -2-(4-acetiloxi-3-metoxifenil)etenil]quinolina (E2), y 2-[(E)-2-(2,3-diacetiloxifenil)etenil]quinolina (E3). Los resultados se expresan como concentración letal media $\left(\mathrm{CL}_{50}\right)$ para la citotoxicidad y concentración efectiva media $\left(\mathrm{CE}_{50}\right)$ para la actividad leishmanicida.

\section{Materiales y métodos}

\section{Síntesis de los compuestos}

Las 2-estirilquinolinas se prepararon a partir de una solución de 8-hidroxiquinaldina (o quinaldina) en anhídrido acético, a la cual se agregó posteriormente el correspondiente aldehído aromático (2,3dihidroxibenzaldehído o vainillina), de acuerdo con la metodología descrita en trabajos previos $(15,19)$.

\section{Preparación de las soluciones}

Para la evaluación de la actividad citotóxica se hicieron seis diluciones dobles seriadas, preparadas a partir de una solución madre de $200 \mu \mathrm{g} / \mathrm{ml}$ en dimetil sulfóxido, y se obtuvieron seis soluciones de concentraciones equivalentes a 100,50, 25, 12,5 y $6,25 \mu \mathrm{g} / \mathrm{ml}$ para las 2-estirilquinolinas sintetizadas.

Para la preparación de las soluciones cuya actividad leishmanicida se evaluaba, se tomaron como referentes los valores de concentración letal media determinados para cada uno de los compuestos estudiados. Para el compuesto E1, se tomaron soluciones de concentraciones equivalentes a $5,6,1,4,0,35$ y $0,0875 \mu \mathrm{g} / \mathrm{ml}$. Para el E2, las concentraciones evaluadas fueron de 68,1, 17,025, 4,25 y $1,06 \mu \mathrm{g} / \mathrm{ml}$, y para el E3, se evaluaron concentraciones de $4,0,1,0,0,25$ y $0,0625 \mu \mathrm{g} / \mathrm{ml}$, todas preparadas en dimetil sulfóxido (DMSO).

Parásito. Para los ensayos de actividad leishmanicida in vitro, se utilizaron promastigotes de L. (V) panamensis (UA140-pIReGFP) cultivados en medio bifásico Novy-MacNeil-Nicolle (NNN) modificado, empleando como fase líquida solución tampón salino de fosfatos (PBS) y glucosa, y se incubaron a $26{ }^{\circ} \mathrm{C}$ para, posteriormente, obtener amastigotes intracelulares.

Células. Estas correspondieron a macrófagos humanos U937 (CRL-1593- 2TM) provenientes del American Type Cell Collection (ATCC), las cuales se mantuvieron cultivadas en suspensión en medio RPMI-1640 con suplemento de suero fetal bovino al $10 \%$, e incubado a $37^{\circ} \mathrm{C}$ con $5 \%$ de $\mathrm{CO}_{2}$.

\section{Actividad citotóxica en las células U937}

La citotoxicidad se evaluó en las células U-937 mediante el micrométodo enzimático con bromuro de 3-(4,5-dimetiltiazol-2-il)-2,5-difeniltetrazolio (MTT). Las células estaban en fase exponencial de crecimiento y se ajustaron a una concentración de 100.000 células $/ \mathrm{ml}$ en platos para cultivo celular de 96 pozos en medio RPMI-1640 y con $10 \%$ de suero fetal bovino. Como control negativo se usaron células bajo las mismas condiciones, pero sin los productos de síntesis, y como control de citotoxicidad, la anfotericina B.

Con las diferentes soluciones de los derivados quinolínicos, tanto las células como los controles se incubaron a $37{ }^{\circ} \mathrm{C}$ en una atmósfera de $5 \%$ de $\mathrm{CO}_{2}$ durante 72 horas. Transcurrido el período de incubación, el efecto de los compuestos en las células se determinó midiendo la actividad de la enzima mitocondrial succinato-deshidrogenasa. Para ello, se añadieron $10 \mu \mathrm{l} /$ pozo de una solución del reactivo de MTT con una concentración de $5 \mu \mathrm{g} /$ $\mathrm{ml}$ y, posteriormente, se incubó a una temperatura de $37{ }^{\circ} \mathrm{C}$ durante tres horas. Pasado este período de incubación, se agregaron $100 \mu \mathrm{l}$ por pozo de una solución de isopropanol al $50 \%$ y de dodecil sulfato de sodio al $10 \%$ para disolver los cristales de formazán (20). La producción de formazán es proporcional al porcentaje de células viables, lo que se midió en un lector de microplacas (BioRad Benchmark $^{\text {TM }}$ ) a una densidad óptica de $570 \mathrm{~nm}$ a los 30 minutos de haber añadido la solución de isopropanol y de dodecil sulfato de sodio. Los ensayos se hicieron dos veces con tres réplicas para cada concentración evaluada y los resultados se expresaron como concentración letal media, calculada por el método Probit de análisis dosisrespuesta (21) con el programa estadístico SAS Data Analysis.

\section{Actividad leishmanicida en Leishmania (Viannia) panamensis}

Para la evaluación de la efectividad de los derivados quinolínicos en los amastigotes intracelulares, se usaron promastigotes de $L$. (V.) panamensis, UA140-pIReGFP, previamente obtenidos a partir de un aspirado de lesión en hámster, con el fin de garantizar una mayor infección de los macrófagos in vitro. Se cultivaron muestras del aspirado de las lesiones producidas por la infección del parásito en tubos de cultivo que contenían medio NNN y se incubaron a una temperatura de $27^{\circ} \mathrm{C}$ hasta obtener promastigotes en fase estacionaria de crecimiento, 
los cuales se emplearon posteriormente para la infección de las células U937 y para obtener los amastigotes intracelulares (22).

Para la infección de las células U-937, se centrifugaron las células cultivadas en suspensión a 1.500 rpm durante 10 minutos y, luego de descartar el sobrenadante, el botón de células se resuspendió en una concentración de $1 \times 10^{5}$ células $/ \mathrm{ml}$ de medio RPMI 1640 al $10 \%$ y suero fetal bovino con $0,1 \mu \mathrm{g} / \mathrm{ml}$ de acetato de forbol miristato. En cada uno de los 24 pozos de una placa para cultivo celular se dispersó $1 \mathrm{ml}$ de la suspensión de células y se incubó a una temperatura de $37^{\circ} \mathrm{C}$ con una atmosfera de $\mathrm{CO}_{2}$ al $5 \%$. Transcurridas 48 horas de incubación, las células se infectaron con promastigotes en fase estacionaria de crecimiento en una proporción de 15:1 entre parásitos y célula. Las placas se incubaron a $34{ }^{\circ} \mathrm{C}$ en un atmósfera de $\mathrm{CO}_{2}$ al $5 \%$ durante dos horas. Después se hicieron dos lavados con PBS para eliminar los parásitos libres, se agregó un medio nuevo y las células se incubaron nuevamente.

Como control de la infección se usaron células infectadas y cultivadas a las que no se añadieron los derivados quinolínicos, y como control de efectividad se empleó la anfotericina B. Las células infectadas se expusieron durante 72 horas a cada una de las concentraciones de los derivados quinolínicos sintetizados. Posteriormente, se removieron cuidadosamente del fondo de la placa utilizando el émbolo de una jeringa y se analizaron por citometría de flujo, lo que dio una lectura de $488 \mathrm{~nm}$ de excitación y 525 nm de emisión con un láser de argón (23).
Cada concentración de los derivados quinolínicos y de los controles se evaluó por triplicado en dos experimentos diferentes. Los resultados se expresaron como concentración efectiva media $\left(\mathrm{CE}_{50}\right)$ y se calcularon mediante el análisis estadístico de regresión lineal Probit empleando el programa CellQuest Pro.

Para establecer la relación entre las actividades citotóxica y leishmanicida, se calculó el índice de selectividad, que corresponde al cociente entre la concentración letal media y la concentración efectiva media $\left(\mathrm{IS}=\mathrm{CL}_{50} / \mathrm{CE}_{50}\right)$.

\section{Resultados}

Los resultados de la evaluación de las actividades leishmanicida y citotóxica de las 2-estirilquinolinas E1, E2 y E3 (figura 1), así como los índices de selectividad calculados, se presentan en el cuadro 1.

Los resultados sugieren que el compuesto E3 es muy activo contra amastigotes intracelulares de $L$. (V) panamensis, con un valor de $\mathrm{CE}_{50}$ de $1,4 \pm 0,1 \mu \mathrm{g} /$ $\mathrm{ml}$, mientras que los compuestos E1 y E2 tuvieron actividad leishmanicida en una concentración mayor que la de su actividad tóxica en la célula. Al comparar la actividad citotóxica con la actividad leishmanicida, se encontró que el compuesto E3 fue más selectivo para eliminar al parásito, ya que su índice de selectividad fue de 3,9, mientras que en los compuestos E1 y E2 fue menor de 1,0.

\section{Discusión}

Las 2-estirilquinolinas conforman un grupo de moléculas que han presentado una gran variedad de actividades biológicas, entre las que se destaca<smiles>CCCCCOc1cccc(C=Cc2ccc3cccc(OC(C)=O)c3n2)c1OC(C)=O</smiles><smiles>COc1cc(/C=C/c2ccc3ccccc3n2)ccc1OC(C)=O</smiles><smiles>CC(=O)Oc1cccc(/C=C/c2ccc3ccccc3n2)c1OC(C)=O</smiles>

Figura 1. Estructura química de las 2-estirilquinolinas E1, E2 y E3 
Cuadro 1. Citotoxicidad, actividad leishmanicida e índice de selectividad de las 2-estirilquinolinas E1, E2 y E3

\begin{tabular}{cccc}
\hline Estirilquinolinas & $\begin{array}{c}\mathrm{CL}_{50}(\mu \mathrm{g} / \mathrm{ml}) \\
\mathbf{X} \pm \mathrm{DE} \\
\text { en U-937 }\end{array}$ & $\begin{array}{c}\mathrm{CE}_{50}(\mu \mathrm{g} / \mathrm{ml}) \\
\mathbf{X} \pm \mathrm{DE} \\
\text { en amastigotes } \\
\text { intracelulares }\end{array}$ & IS \\
\hline E1 & $5,6 \pm 0,3$ & $>5,6$ & $<1,0$ \\
E2 & $68,1 \pm 11,3$ & $>68,1$ & $<1,0$ \\
E3 & $5,4 \pm 1,1$ & $1,4 \pm 0,1$ & 3,9 \\
Anfotericina B & $51,4 \pm 2,8$ & $0,05 \pm 0,01$ & 1.028 \\
\hline
\end{tabular}

$\mathrm{CE}_{50}$ : concentración efectiva media; $\mathrm{CL}_{50}$ : concentración letal media; IS: índice de selectividad $=\mathrm{CL}_{50} / \mathrm{CE}_{50}$

la actividad contra los protozoos $(15,24)$. Diversos autores han reportado la actividad leishmanicida de estas moléculas. Loiseau, et al., evaluaron la actividad leishmanicida in vitro contra amastigotes de $L$. donovani y determinaron que el compuesto 2-[(E)-2-(3,4-diacetiloxifenil)etenil]quinolin-8-ilacetato arrojaba valores de concentración de inhibición media $\left(\mathrm{IC}_{50}\right)$ de 13,5 $\mu \mathrm{M}(16)$.

Por otra parte, Franck, et al., evaluaron la actividad leishmanicida de varias quinolinas 2 sustituidas contra $L$. amazonensis y $L$. infantum, y calcularon para la estirilquinolina 2-[(E)-2-(3,4-dimetoxifenil) etenil]quinolina un valor de $\mathrm{IC}_{50}$ de $22 \mu \mathrm{M}$ en $L$. amazonensis, y en $L$. infantum, un valor de $I_{50}$ mayor de $32 \mu \mathrm{M}$ (12). Estos resultados ratifican que las 2-estirilquinolinas son compuestos activos frente a diferentes especies de Leishmania y que las variaciones en el tipo de los elementos que sustituyen y en las posiciones en su estructura química, ya sea en el núcleo quinolínico o en el anillo aromático, afectan la actividad de los compuestos frente al parásito.

Mesa, et al., evaluaron la actividad leishmanicida in vitro de las estirilquinolinas 2-[(E)-2-(2,5-dimetoxifenil) etenil]quinolina y 2-[(E)-2-(2,3-dimetoxifenil)etenil] quinolina y $\mathrm{N}$-\{4-[(E)-2-quinolin-2-iletenil]fenil\} acetamida contra amastigotes intracelulares de $L$. (Viannia) panamensis, y calcularon valores de $\mathrm{CE}_{50}$ de $1,4,1,8$ y $1,7 \mu \mathrm{g} / \mathrm{ml}$, respectivamente; en cuanto a la toxicidad frente a la línea celular U937, reportaron valores de $\mathrm{CL}_{50}$ de 3,7, 6,2 y $4,5 \mu \mathrm{g} / \mathrm{ml}$, con índices de selectividad de 1, 1,2 y 0,2 , respectivamente (15).

En este trabajo se reportan los resultados de citotoxicidad y actividad leishmanicida en términos de la $C L_{50}$ y la $C E_{50}$ para tres 2-estirilquinolinas (figura 1). Los resultados obtenidos sugieren que la ubicación de un grupo metoxilo sobre el anillo aromático del compuesto E2 produce una disminución sustancial de su toxicidad cuando la $\mathrm{CL}_{50}$ es de $68,1 \mu \mathrm{g} / \mathrm{ml}$, comportamiento que se evidencia al comparar los mismos valores obtenidos para las 2-arilquinolinas E1 y E3, los cuales fueron de 5,6 y $5,4 \mu \mathrm{g} / \mathrm{ml}$, respectivamente. Por lo tanto, en este tipo de moléculas la presencia de sustituyentes del tipo acetil aumenta notablemente la toxicidad. Por el contrario, los grupos metoxilo inducen una variación en la toxicidad hasta valores inclusive menores que los de la anfotericina $B$.

En general, en este trabajo los resultados relativos a la citotoxicidad (cuadro 1) concuerdan con los encontrados por Mesa, et al., quienes informan que entre los compuestos evaluados, los que presentaron menor toxicidad fueron los que carecen de sustitución en la posición 8 del anillo quinolínico, situación que es similar en el E2, y subrayan que la naturaleza química de los sustituyentes en el sistema quinolínico y en el anillo aromático influyeron directamente en la toxicidad del compuesto.

En cuanto a los resultados de la actividad leishmanicida, los datos relativos al E1 (cuadro 1) sugieren que este compuesto no tiene potencial contra $L$. (V.) panamensis. Sin embargo, Loiseau, et al., reportan la actividad de la 2-estirilquinolina 2-[(E)-2-(3,4-diacetiloxifenil)etenil]quinolin-8-ilacetato contra $L$. donovani (16). Esta molécula se diferencia del compuesto E1 en la posición de los grupos acetil en el anillo aromático. Por otra parte, los datos sobre la actividad leishmanicida de E2 y E3 sugieren que el cambio de un grupo acetilo por uno metoxilo en el anillo aromático desfavorece la actividad contra $L$. (V.) panamensis, lo cual se refleja en los valores de $C_{50}$ en estos dos compuestos, que corresponden a $>68,1$ y $1,4 \mu \mathrm{g} /$ $\mathrm{ml}$, respectivamente.

Con relación a la actividad leishmanicida, los resultados obtenidos permiten destacar como promisorio el compuesto E3, el cual se caracteriza estructuralmente por presentar dos grupos acetil en el anillo aromático y por la ausencia de sustituyentes en la posición 8 del núcleo quinolínico, en comparación con E1 y E2. Estos resultados concuerdan con trabajos previos (15).

En la estrategia diseñada para la identificación de compuestos candidatos a convertirse en medicamentos, los investigadores han determinado como punto de referencia valores de $\mathrm{CE}_{50} \leq 10 \mu \mathrm{g} / \mathrm{ml}$, concentración en la cual un compuesto se considera muy activo. Por lo tanto, de los tres compuestos bajo evaluación, la estirilquinolina E3 se considera como un candidato promisorio para el desarrollo 
de medicamentos, toda vez que su capacidad leishmanicida supera la capacidad citotóxica, lo que se evidenció en una $\mathrm{CE}_{50}$ de $1,4 \mu \mathrm{g} / \mathrm{ml}$ y una $\mathrm{CL}_{50}$ de $5,1 \mu \mathrm{g} / \mathrm{ml}$. Además, se considera que una sustancia tiene actividad leishmanicida promisoria cuando presenta un índice de selectividad mayor de 1, porque dicho valor está en correlación con un valor de $\mathrm{CL}_{50}$ alto (lo que significa que el compuesto tiene un bajo potencial citotóxico), y con un valor de $\mathrm{CE}_{50}$ bajo (lo que significa que el compuesto tiene un alto potencial de actividad leishmanicida).

Sin embargo, los valores de los índices de selectividad no siempre se ajustan a este criterio, ya que es posible encontrar sustancias muy tóxicas y muy activas, en las cuales también es posible obtener índices de selectividad mayores de 1 (25). Este es el caso del compuesto E3, el cual es muy activo contra el parásito, a pesar de ser muy citotóxico. En este caso, el índice de selectividad de 3,9 mostrado por el compuesto E3 sugiere que, para producir un efecto nocivo contra el parásito, este compuesto requiere cerca de cuatro veces menos cantidad de la necesaria para dañar la célula huésped. Este hecho demuestra, además, que el daño observado en el parásito intracelular no se debe a la muerte de la célula huésped. Dicho comportamiento constituye un importante punto de partida para considerar a E3 como un compuesto promisorio, ya que existen mecanismos in vivo que permiten reducir de manera significativa la toxicidad, ya sea con liposomas o con polímeros que propicien la reducción de la toxicidad celular sin que ello implique pérdida de la eficacia terapéutica (26). Por su parte, los compuestos E1 y E2, cuyos valores de $\mathrm{CE}_{50}$ fueron superiores a los de la $\mathrm{CL}_{50}$ y, por lo tanto presentaron un índice de selectividad menor de 1, son poco prometedores como agentes leishmanicidas.

En conclusión, la síntesis de compuestos orgánicos constituye una herramienta importante en la búsqueda de aquellos que puedan convertirse en el principio activo de medicamentos para el tratamiento de las diferentes formas clínicas de la leishmaniasis. Los valores de $\mathrm{CE}_{50}$ para las tres estirilquinolinas evaluadas, muestran que solo una de ellas (E3) tiene potencial contra los amastigotes intracelulares de $L$. (V.) panamensis, mientras que, a pesar de su baja toxicidad, E1 y E2 no mostraron potencial de actividad contra el parásito.

Este trabajo contribuye a la búsqueda de nuevas moléculas activas contra $L$. ( $V$.) panamensis al proponer una variación en las posiciones y en la clase de sustituyentes ya reportados por diversos autores, demostrando que la variación estructural propuesta para la 2-estirilquinolina E3 es efectiva en la cepa de Leishmania bajo evaluación. Por otra parte, puesto que se ha informado que las 2-estirilquinolinas presentan actividad frente a diferentes tipos de protozoos, resultaría interesante evaluar la actividad de estos compuestos contra los agentes causantes de la malaria y la enfermedad de Chagas.

\section{Conflicto de intereses}

Los autores de este trabajo declaramos que no existe conflicto de intereses.

\section{Agradecimientos}

A la Universidad de Córdoba, por la financiación de la investigación, y al Programa de Estudio y Control de Enfermedades Tropicales, PECET, de la Universidad de Antioquia, por su colaboración en la realización de los ensayos de actividad biológica.

\section{Referencias}

1. Ríos JM, Sousa O. Inmunología en la infección por Leishmania: conceptos actuales. Revista Médico-Científica. 2010;3:19-31.

2. Montalvo AM. Leishmaniasis. Aspectos de interés sobre un parasitismo exótico para Cuba. Revista Cubana de Higiene y Epidemiología. 2010;48:78-92.

3. Umakant S, Sarman S. Insect vectors of Leishmania: Distribution, physiology and their control. J Vector Borne Dis. 2008;45:255-72.

4. Vásquez L. Terapéutica antileishmania: revisando el pasado, el presente y el futuro. Gac Méd Caracas. 2009;117:93-111.

5. Gamboa Y, Delgado G. Estudio de la infección de Leishmania del complejo Viannia mediante citometría de flujo y coloración de Giemsa empleando líneas de macrófagos humanos y murinos (U-937 y J-774). Revista Colombiana de Ciencias Químico-Farmacéuticas. 2008;37:84-95.

6. World Health Organization. Control of the leishmaniases: Report of a meeting of the WHO Expert Committee on the Control of Leishmaniases. WHO Technical Report Series 949. Geneva: World Health Organization; 2010. p. 186. Fecha de consulta: 27 de junio de 2014. Disponible en: http://whqlibdoc.who.int/trs/WHO_TRS_949_eng.pdf.

7. Instituto Nacional de Salud. Dirección de Vigilancia y Análisis de Riesgo en Salud Pública. Boletín Epidemiológico Semanal, BES. Semana epidemiológica número 22 de 2014, p. 21-3. Fecha de consulta: 27 de junio de 2014. Disponible en: http://ins.gov.co/boletin-epidemiologico/Boletn\%20 Epidemiolgico/2014\%20Boletin\%20epidemiologico\%20 semana\%2022.pdf? Mobile=1\&Source $=\% 2$ Fboletinepidemiologico \%2F_layouts\%2Fmobile\%2Fmblwp.asp.

8. Instituto Nacional de Salud. Leishmaniasis. Protocolo de Vigilancia en Salud Pública. Leishmaniasis. 2014. Fecha de consulta: 28 de junio de 2014. Disponible en: http://www. ins.gov.co/lineas-de-accion/Subdireccion-Vigilancia/sivigila/ Protocolos\%20SIVIGILA/PRO\%20Leishmaniasis.pdf. 
9. Olliaro P, Sundar S. Anthropometrically-derived dosing and drug costing calculations for treating visceral leishmaniasis in Bihar. Trop Med Int Health. 2009;14:88-92. http://dx.doi. org/10.1111/j.1365-3156.2008.02195x

10. López L, Cruz C, Godoy G, Robledo SM, Vélez ID. Thermotherapy effective and safer than miltefosine in the treatment of cutaneous leishmaniasis in Colombia. Rev Inst Med Trop Sao Paulo. 2013;55:197-204. http://dx.doi. org/10.1590/S0036-46652013000300011

11. Kouznetsov V, Meléndez C. Búsqueda de nuevos agentes antiprotozoarios selectivos. Revista Medunab. 2009;12:3345.

12. Franck X, Fournet A, Prina E, Mahieux R, Hocquemiller R, Figadere B. Biological evaluation of substituted quinolines. Bioorg Med Chem Lett. 2004;14:3635-8. http://dx.doi.org/10. 1016/j.bmcl.2004.05.026

13. Fakhfakh MA, Fournet A, Prina E, Mouscadet JF, Franck $\mathbf{X}$, Hocquemiller $\mathbf{R}$, et al. Synthesis and biological evaluation of substituted quinolines: Potential treatment of protozoal and retroviral co-infections. Bioorg Med Chem. 2003;11:501323. http://dx.doi.org/10.1016/j.bmc.2003.09.007

14. Nakayama H, Loiseau PM, Bories C, Torres S, Schinini A Serna E, et al. Efficacy of orally administered 2-substituted quinolines in experimental murine cutaneous and visceral leishmaniases. Antimicrob. Agents Chemother. 2005;49:495056. http://dx.doi.org/10.1128/AAC.49.12.4950-4956.2005

15. Mesa AM, Molano PA, Seón B, Figadere B, Robledo SM, Muñoz D, et al. Síntesis y actividades leishmanicida y citotóxica in vitro de análogos 2-arilquinolinas. VITAE. 2008;15:259-66.

16. Loiseau P, Gupta S, Verma A, Srivastava S, Puri SK, Sliman $\mathbf{F}$, et al. In vitro activities of new 2-substituted quinolines against Leishmania donovani. Antimicrob Agents Chemother. 2011;55:1777-80. http://dx.doi.org/10.1128/AAC. 01299-10

17. Leonard JT, Roy K. Exploring molecular shape analysis of styrylquinoline derivatives as HIV-1 integrase inhibitors. Eur
J Med Chem. 2008;43:81-92. http://dx.doi.org/10.1016/j. ejmech.2007.02.021

18. Walaa O, Osmo H. Synthesis of 4-(2-arylvinyl)-8-hydroxyquinolines via anhydrous Heck coupling reaction and the $\mathrm{PL}$ properties of their Al complexes. Tetrahedron. 2009; 65:4422-8. http://dx.doi.org/10.1016/j.tet.2009.03.028

19. Martínez M, Giménez C, Gómez A, Franck X, Fournet A, Hocquemiller $\mathbf{R}$, et al. Nematocidal and trichomonacidal activities of 2-substituted quinolines. Farmaco. 2005;60:21924. http://dx.doi.org/10.1016/j.farmac.2004.11.010

20. Weniger B, Robledo SM, Arango GJ, Deharo E, Aragón R, Muñoz V, et al. Antiprotozoal activities of Colombian plants. J Ethnopharmacol. 2001;78:193-200. http://dx.doi. org/10.1016/S0378-8741(01)00346-4

21. Finney JD. Statistical logic in the monitoring of reactions to therapeutic drugs. Methods Inf Med. 1971;10:237-45.

22. Cardona WC, Robledo SM, Rojano BA, Alzate CF, Muñoz DL, Sáez J. Actividad leishmanicida y antioxidante de extractos de Piper daniel-gonzalezii Trel. (Piperaceae). Revista Cubana de Plantas Medicinales. 2013;18:268-77.

23. Ríos YK, Otero AC, Muñoz DL, Echeverry M, Robledo SM, Yépez MA. Actividad citotóxica y leishmanicida in vitro del aceite esencial de manzanilla (Matricaria chamomilla). Revista Colombiana de Ciencias Químico Farmacéuticas. 2008;37:200-11.

24. García P, Genes C, Molano P, Torres O, Sáez J, Triana O. Evaluation of the trypanocidal, cytotoxic and genotoxic activity of styrylquinoline analogs. J Chemother. 2010;22:3944. http://dx.doi.org/10.1179/joc.2010.22.3.169

25. Cardona DP. Moléculas bioactivas contra Leishmania (Viannia) panamensis. Actividad y optimización molecular (tesis). Medellín: Universidad de Antioquia; 2006.

26. Miñonez J. Anfotericina liposomal: una aproximación fisicoquímica al conocimiento de su mecanismo de acción. Anales de la Real Academia Nacional de Farmacia. 2002;68:2-17. 